\title{
The adherens junction-associated protein 1 is a negative transcriptional regulator of MAGEA2, which potentiates temozolomide-induced apoptosis in GBM
}

\author{
LIANG ZENG ${ }^{1,3,4}$, CHUNSHENG KANG ${ }^{3,4}$, CHUNHUI DI $^{3,4}$, BRIAN E. FEE ${ }^{2}$, \\ MIRIAM RIVAS ${ }^{2}$, JAMES LIN ${ }^{3,4}$ and DAVID CORY ADAMSON ${ }^{2,3,4}$ \\ ${ }^{1}$ Department of Neurosurgery, Tongji Hospital, Huazhong University of Science and Technology, Wuhan, P.R. China; \\ ${ }^{2}$ Durham VA Medical Center; ${ }^{3}$ Preston Robert Tisch Brain Tumor Center, Departments of Surgery and Neurobiology; \\ ${ }^{4}$ Duke University Medical Center, Durham, NC, USA
}

Received September 15, 2013; Accepted October 21, 2013

DOI: 10.3892/ijo.2014.2277

\begin{abstract}
Previous studies identified the frequent loss of adherens junction-associated protein 1 (AJAP1) expression in glioblastoma (GBM) and its correlation with worse survival. AJAP1 may suppress glioma cell migration, which plays an important role in tumor progression in malignant gliomas such as GBM. However, the role of AJAP1 in cell cycle arrest or apoptosis and resistance to chemotherapy remains unclear. Based on microarray screening results, quantitative PCR and luciferase plasmid reporter constructs were used to evaluate the possible regulatory role of AJAP1 on MAGEA2 expression and function. Cell death assays, TUNEL and other markers of apoptosis were utilized to detect cell apoptosis. Restoration of AJAP1 expression in glioma cells was analyzed after temozolomide exposure. AJAP1 suppressed the expression of MAGEA2 and inhibited the transcriptional activity of MAGEA2 in glioma cells. As AJAP1 expression decreased MAGEA2 protein expression apoptosis increased moderately. Consistent with increased cell death, the induced loss of MAGEA2 expression correlated with increased caspase $3 / 7$ activity, BCL2/BAX ratio and TUNEL signal. AJAP1 expression enhanced cell death in the presence of temozolomide. This study suggests AJAP1 may also function as a pro-apoptotic factor and potentiate cell death by temozolomide in glioma cells. This effect may be partially explained by AJAP1-mediated gene regulation of MAGEA2.
\end{abstract}

\section{Introduction}

Glioblastoma multiforme (GBM) remains one of the most common and biologically aggressive tumors in adults. Despite

Correspondence to: Professor David Cory Adamson, Duke University Medical Center, Department of Surgery, DUMC Box 2624, 421-MSRB Bldg., Research Drive, Durham, NC 27710, USA

E-mail: cory.adamson@duke.edu

Key words: adherens junction-associated protein-1, glioblastoma, migration, MAGEA2, gene regulation, apoptosis standard therapeutic protocols, which include maximal surgical resection followed by regional fractionated radiation and chemotherapy with temozolomide, the prognosis of patients with GBM remains dismal, with median survival of less than 15 months (1). As they are invariably fatal due, in part, to resistance to postsurgical therapy, identification of genetic alterations which can predict efficient management and areas of targets for therapy are continuously being sought $(2,3)$.

A broad range of human malignancies is associated with non-random 1p36 deletions, suggesting the existence of tumor suppressors encoded in this region (4). Allelic loss involving $1 \mathrm{p} 36$ is a common chromosomal aberration observed in oligodendroglioma and has been associated with radiation and chemosensitivity, as well as enhanced tumor infiltration (5). We recently discovered the frequent loss of expression of adherens junction-associated protein 1 (AJAP1) in GBM via multiple genome-wide studies. Located on 1p36, AJAP-1 may have tumor suppressor-like qualities and may play an important role in regulation of cell migration and adhesion (6). However, the role of AJAP1 in cell cycle arrest or apoptosis and resistance to chemotherapy remain largely unexplored.

Temozolomide (TMZ) is the first-line chemotherapeutic drug agent for GBM with excellent CNS bioavailability $(7,8)$. It is an alkylating cytostatic drug, which causes DNA damage and induces cell death. However, clinical response toward TMZ is generally short-lived, possibly due to multiple factors such as tumor cell heterogeneity, resistant glioma stem cells, rapid transformation, genetic instability and selective pressures $(9,10)$. The available data suggest that neither MGMT protein expression levels nor MGMT promoter methylation status is a sufficiently accurate predictor of the sensitivity of the glioma cells to TMZ (11). More recently, further studies indicated that additional pathways, such as activation of DNA damage checkpoint responses and the changes in mitochondria DNA and electron transport chain may contribute to TMZ resistance $(12,13)$. Therefore, the intrinsic and acquired resistance towards TMZ makes it crucial to find new therapeutic strategies aimed at improving the prognosis of patients suffering from GBM. In the present study the concomitant treatment of GBM cells with AJAP1-restoration and TMZ in overcoming TMZ resistance was investigated. 
MAGEA2 protein induces a novel p53 inhibitory loop involving recruitment of histone deacetylase 3 (HDAC3) to a MAGEA2-p53 complex (14). This complex formation strongly downregulates the p53 transactivation function in melanoma without changing p53 expression levels (15). Other data suggest potential involvement of MAGEA family proteins in modulating cell survival (16). The correlation between MAGEA2 expression and resistance to apoptosis has been validated in various kinds of tumors partly through its interaction with the p53 pathway, where high MAGEA2 levels reestablish p53 response and increase chemoresistance (17-19). In this study, we investigated AJAP1 function in the induction of cell apoptosis and potentiation of TMZ-induced apoptosis in glioma cells through the transcriptional downregulation of MAGEA2.

\section{Materials and methods}

Cell culture and drug treatments. HEK293, U87MG, U251MG, D373MG and D409MG cells were maintained in DMEM - High Glucose medium (Gibco, Carlsbad, CA, USA) supplemented with $10 \%$ FBS (Gibco) and incubated in a humidified incubator at $37^{\circ} \mathrm{C}$ with $5 \% \mathrm{CO}_{2}$. U87MG, U251MG, D373MG and D409MG cells were previously stably transfected with the GFP-AJAP1 plasmid (6). Similar cells were also transfected with GFP-N1 vector using identical transfection techniques to be used as a control. Clones were maintained in $100 \mu \mathrm{g} / \mathrm{ml}$ geneticin. For temozolomide (TMZ) studies, glioma cells were seeded in $10 \mathrm{~cm}$ round Petri dishes, 24- or 96-well plates, allowed to attach overnight, then treated with $100 \mu \mathrm{M}$ temozolomide (TMZ, Sigma-Aldrich Co., St. Louis, MO, USA) for $48 \mathrm{~h}$. In control groups, the same concentration of dimethyl sulfoxide (DMSO) was used to treat cells. Fresh drug and medium were changed every $24 \mathrm{~h}$.

RNA preparation and GeneChip array assay. GBM cell lines (D373) stably expressing GFP-N1 (vector control) or GFP-AJAP1 were assessed for their gene expression profile using a high-density oligonucleotide array (GeneChip Human Genome U133 Plus 2.0 Array; Affymetrix, Santa Clara, CA, USA). Total RNA was extracted from freshly prepared confluent cells. cDNA was generated from the total RNA using Qiagen's RNeasy system (Quiagen, Valencia, CA, USA), then fragmented and hybridized to a DNA oligonucleotide expression array (Affymetrix Human Genome U133A 2.0 Array). The hybridized probe array was washed and stained following the manufacturer's instructions. The probe array was then scanned with a confocal laser scanner (GeneChip Scanner 3000; Affymetrix). The expression array analysis for each cell line was run in triplicate. The expression level of each gene $(22,277$ probe sets for approximately 14,500 human genes) was calculated to identify discriminative genes by Partek (St. Louis, MO, USA).

Real-time quantitative RT-PCR. Quantification of ten candidate genes was performed to confirm Affymetrix data by the real-time quantitative reverse transcription-PCR method. Total RNA was extracted from GBM cell lines as described previously. The real-time PCR reaction mixture was prepared using a TaqMan Universal Master Mix (Applied Biosystems, Foster City, CA, USA), consisting of $120 \mathrm{nM}$ of primer, $200 \mathrm{nM}$ probes, and $2.5 \mu \mathrm{l}$ of cDNA sample. PCR conditions were as follows: $95^{\circ} \mathrm{C}$ for $10 \mathrm{~min} ; 45$ cycles at $95^{\circ} \mathrm{C}$ for $30 \mathrm{sec}, 62^{\circ} \mathrm{C}$ for $30 \mathrm{sec}$, and $72^{\circ} \mathrm{C}$ for $30 \mathrm{sec}$, then $72^{\circ} \mathrm{C}$ for $1 \mathrm{~min}$. Each reaction was run in triplicate, and we generated a standard curve for each probe using serial dilutions from reference cDNA to quantify relative gene expression. The glyceraldehyde-3-phosphate dehydrogenase (GAPDH) gene was used as an endogenous reference, and each sample was normalized to its GAPDH content.

Isolation of the proximal promoter of the human MAGEA2 gene and plasmid construction. The region of the human MAGEA2 gene 986 bp upstream $(-896$ to +90$)$, containing the complete proximal promoter regulatory elements, was amplified by PCR from genomic DNA. The primer pairs were MAGEA2 5'-GGG GTA CC accaaaggtttgccaagtgtag-3' with a KpnI site at the 5'-end and MAGEA2 5'-CCC AAG CTT ctgccetggtcacacaaag-3' with a HindIII site at the $5^{\prime}$-end. The PCR was conducted at $95^{\circ} \mathrm{C}$ for $1 \mathrm{~min}$ followed by 30 cycles at $95^{\circ} \mathrm{C}$ for $30 \mathrm{sec}, 58^{\circ} \mathrm{C}$ for $1.5 \mathrm{~min}$, $72^{\circ} \mathrm{C}$ for $1 \mathrm{~min}$. The amplified fragment was isolated and purified following agarose electrophoresis using a Gel Extract Kit (Thermo Scientific, Rockford, IL, USA), digested with KpnI and HindIII (New England Biolabs, Ipswich, MA, USA), and ligated into the equivalent sites of the pGL3-enhancer vector (Promega, Madison, WI, USA) to generate the pGL3-MAGEA2 construct. The resulting construct was confirmed by restriction enzyme analysis and DNA sequence analysis.

Transient transfection. For the promoter activity assay,HEK293, U87 and U251 cells were transfected with lipofectamine 2000 (Invitrogen, Carlsbad, CA, USA) in 24-well plates. Each well included $0.5 \times 10^{5}$ cells, $1.0 \mu \mathrm{g}$ pGL3-MAGEA2, $1 \mathrm{ng}$ internal control vector pRL-CMV (Promega), $2 \mu \mathrm{l}$ lipofectamine 2000 and $500 \mu 1$ Opti-MEM I reduced serum media (Gibco). For co-transfection experiments of the GFP-AJAP1 with pGL3-MAGEA2 plasmid, HEK293 cells were transfected with lipofectamine 2000 in 24-well plates, and each well included $0.5 \times 10^{5}$ cells, $0.5 \mu \mathrm{g}$ pGL3-MAGEA2, $0.5 \mu \mathrm{g}$ GFP-AJAP1, $1 \mathrm{ng}$ pRL-CMV, $2 \mu$ l lipofectamine 2000 and $500 \mu$ l Opti-MEM I reduced serum media. Empty pGL3-enhancer vector and GFP-N1 vector were used as control.

Dual-luciferase reporter assays. Twenty-four hours after transfection, the activities of firefly luciferase in pGL3-constructs and Renilla luciferase in pRL-CMV were determined by the dualluciferase reporter assays following manufacturer's protocol (Promega). The cells were rinsed with PBS, and lysed with $1 \mathrm{X}$ passive lysis buffer. A total of $20 \mu 1$ of cell lysate was transferred into the luminometer plates containing $100 \mu 1$ luciferase assay reagent II. Firefly luciferase activity was measured first, and then Renilla luciferase activity was measured after the addition of $100 \mu \mathrm{l}$ Stop \& Glo Reagent. Results were expressed as a ratio of firefly luciferase activity to Renilla luciferase activity.

Western blot analysis. Cultured cells were harvested by scraping in ice-cold PBS and spun down at 2,500 rpm in a $4^{\circ} \mathrm{C}$ centrifuge for $5 \mathrm{~min}$. Cell pellets were then lysed in lysis buffer (25 mM Tris pH 7.4, $150 \mathrm{mM} \mathrm{NaCl,} \mathrm{1 \%} \mathrm{NP-40,} 1$ mM EDTA, $5 \%$ glycerol) containing $1 \%$ of protease inhibitor cocktail (Thermo Scientific). Protein concentration was determined by standard BCA assay (Pierce, Thermo Scientific), and total cell lysates were separated by electrophoresis $10 \%$ precast poly- 
Table I. Summary of fold change of gene expression level between AJAP1 cells (DS) and vector cells (DV) by microarray screening.

\begin{tabular}{|c|c|c|}
\hline Gene title & Gene symbol & $\begin{array}{l}\text { Fold change } \\
\text { (DS vs. DV) }\end{array}$ \\
\hline Melanoma antigen family A, 2///2B & MAGEA2///2B & -116.93 \\
\hline Melanoma antigen family A, 3 & MAGEA3 & -111.46 \\
\hline Proteolipid protein 1 & PLP1 & -37.40 \\
\hline Attractin-like 1 & ATRNL1 & -27.24 \\
\hline Butyrylcholinesterase & BCHE & -25.53 \\
\hline MACRO domain containing 2 & MACROD2 & -19.80 \\
\hline Hypothetical protein LOC169834 & LOC169834 & -17.40 \\
\hline KIAA1239 & KIAA1239 & -15.41 \\
\hline $\begin{array}{l}\text { ADAM metallopeptidase with } \\
\text { thrombospondin type } 1 \text { mmotif, } 3\end{array}$ & $\operatorname{ADAMTS3}(3)(7)(10)$ & -12.95 \\
\hline Tetraspanin 8 & TSPAN8 & -10.73 \\
\hline $\begin{array}{l}\text { Protein kinase, AMP-activated, } \\
\text { alpha } 2 \text { catalytic subunit }\end{array}$ & PRKAA2(6) & 12.30 \\
\hline MARCKS-like 1 & MARCKSL1 & 12.89 \\
\hline Secreted frizzled-related protein 1 & SFRP1 & 14.10 \\
\hline Fibulin 1 & FBLN1(3) & 14.27 \\
\hline Secreted frizzled-related protein 1 & SFRP1 & 17.63 \\
\hline Paraneoplastic antigen MA2 & PNMA2 & 19.78 \\
\hline Neurofascin homolog (chicken) & NFASC(2) & 21.93 \\
\hline $\begin{array}{l}\text { Protein kinase, AMP-activated, } \\
\text { alpha } 2 \text { catalytic subunit }\end{array}$ & PRKAA2 & 22.64 \\
\hline Kynureninase (L-kynurenine hydrolase) & KYNU & 24.08 \\
\hline Docking protein 5 & DOK5 & 24.52 \\
\hline Peptidase inhibitor 15 & PI15 & 29.53 \\
\hline Adherens junctions associated protein 1 & AJAP1 & 40.79 \\
\hline Transmembrane protein 47 & TMEM47 & 106.87 \\
\hline
\end{tabular}

Microarray-based gene expression analysis of D373 GBM cells cultures identified a set of novel candidate genes being upregulated or downregulated relative to AJAP1 restoration.

acrylamide gel (Bio-Rad, Hercules, CA, USA). Gel loading was normalized for equal GAPDH ( $25 \mu \mathrm{g}$ protein per lane). Proteins were then transferred onto Hybond-P polyvinylidene difluoride membranes (Amersham Biosciences, Piscataway, NJ, USA), and the membranes were probed with each antibody. Rabbit polyclonal anti-MAGEA2 (1:100), rabbit monoclonal anti-Bcl2 (1:2000) and rabbit polyclonal anti-Bax $(1: 1,000)$ were obtained from Abcam Biotechnology. Rabbit polyclonal anti-AJAP1 (1:2000) was obtained from Sigma-Aldrich Co. Rabbit monoclonal anti-GAPDH $(1: 1,000)$ and chicken anti-rabbit IgG-HRP $(1: 2,000)$ were purchased from Santa Cruz Biotechnology (Santa Cruz, CA, USA). Immunoblots were detected using a Supersignal West Femto substrate kit (Thermo Scientific) and visualized by autoradiography.

Caspase 3/7 activity assay. A total of $10^{4} \mathrm{U} 87 \mathrm{MG}$ cells and U251 MG cells overexpressing AJAP1 or control (GFP-N1) were seeded into 96-well plates overnight. Room temperature Caspase-Glo 3/7 Reagent (100 $\mu$ l) was added to each well of a white-walled 96-well plate containing $100 \mu \mathrm{l}$ of blank,
DMSO (negative control) cells or TMZ treated cells in culture medium. Wells were gently mixed on a plate shaker at $500 \mathrm{rpm}$ for $30 \mathrm{sec}$, incubated at room temperature for $1 \mathrm{~h}$, then luminescence measured in a plate-reading luminometer.

Confocal TUNEL assay. A total of $10^{5}$ cells were seeded onto 12-mm poly-L-lysine coated coverslips placed in the bottom of $24-w e l l$ plates. After treatment, cells were fixed with $4 \%$ paraformaldehyde for $1 \mathrm{~h}$ at room temperature. After incubating in $0.1 \%$ Triton X-100 for 2 min on ice, a terminal deoxynucleotidyl transferase-mediated dUTP nick-end labelling (TUNEL) reaction solution containing TMR red-labeled nucleotides (Roche, Mannheim, Germany) was added. Transferase was excluded in identical mixtures as negative control. Slides were placed in a humidified atmosphere overnight at $4^{\circ} \mathrm{C}$ in the dark, rinsed with PBS three times, then cover slips were mounted in mounting medium with counterstaining cell nuclei using 4',6-diamidino-2-phenylindole (DAPI) (Vector Laboratories, Burlingame, CA, USA) overnight. Using an excitation wavelength in $540 \mathrm{~nm}$ and detection in $620 \mathrm{~nm}$, confocal images 
A

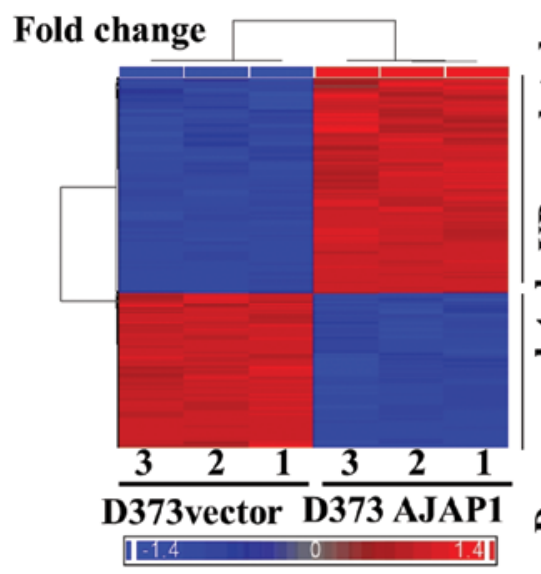

B

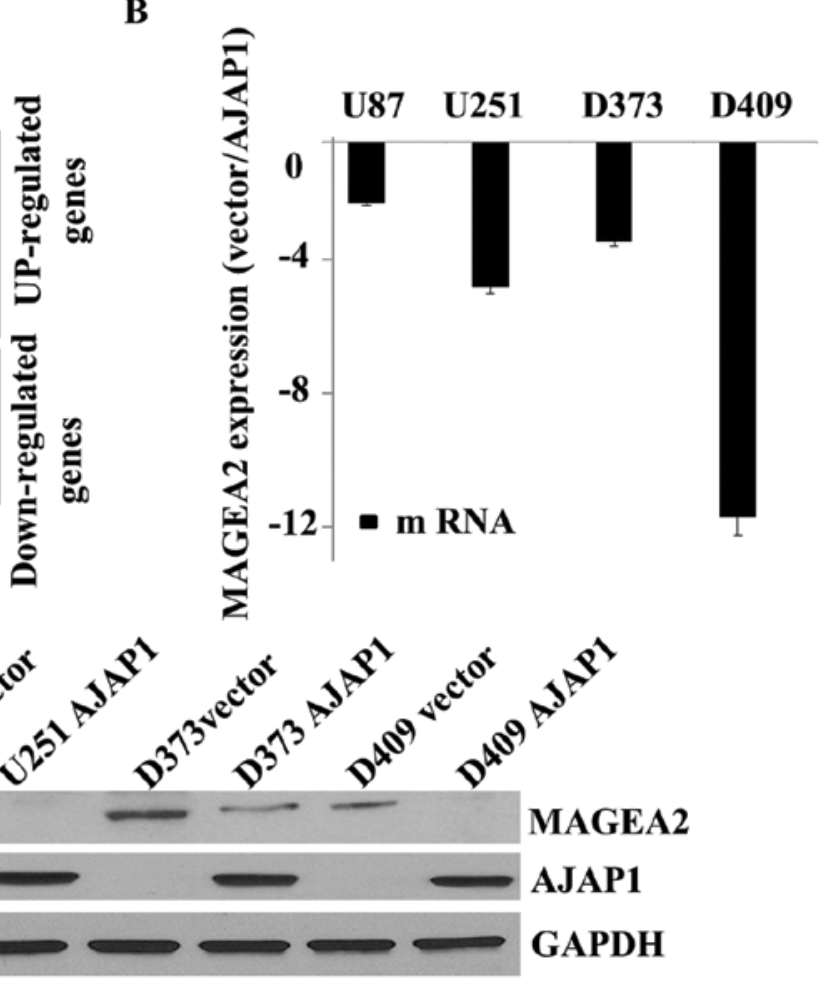

Figure 1. Downregulation of MAGEA2 by AJAP1 restoration. U87, U251, D373MG and D409MG cells were stably transfected with EGFP-AJAP1/GFP-N1 plasmid. (A) Microarray-based gene expression analysis of genome to identify candidate genes being upregulated or downregulated following AJAP1 overexpression by Affymetric GeneChip Human Genome U133 Plus 2.0 Array in EGFP-N1 (vector control) and EGFP-AJAP1 stably transfected D373MG cells. (B) The fold change of mRNA levels for MAGEA2 in 4 pairs of vector/AJAP1 cells were measured by real-time PCR using specific primers and normalized to the housekeeping gene glyceraldehyde-3-phosphate dehydrogenase (GAPDH). Histogram represents the mean of fold change of mRNA expression in samples measured in triplicate for 3 separate subcultures. (C) Representative western blot analysis (done in triplicate) for MAGEA2 and AJAP1 from $25 \mu \mathrm{g}$ of whole cell lysates with EGFP-AJAP1 transfected GBM cells or control (EGFP- N1). GAPDH demonstrates equal protein loading.

were taken with a microscope (Zeiss 780 upright confocal; Carl Zeiss, Göttingen, Germany). Images were collected using the Laser-Sharp 2000 software.

Statistical analysis. All experiments were done in triplicate. Statistical significance was set at $\mathrm{P}<0.05$. Analyses were done with Microsoft Excel and SAS E-guide statistical packages (SAS Inc., Cary, NC, USA).

\section{Results}

Identification of AJAP1-associated gene changes that may augment TMZ-induced cytotoxicity. To investigate the possible transcriptional regulatory role of AJAP1, microarray-based gene expression analysis of GBM cell lines (D373) stably expressing EGFP-N1 (vector control) or EGFP-AJAP1 identified a set of novel candidate genes being upregulated or downregulated following AJAP1 overexpression (Fig. 1A). MAGEA2 was identified as a likely candidate that may alter cytotoxicity responses. The fold change in MAGEA2 (116.931fold decrease) was one of the most significant in the genome after AJAP1 overexpression (Table I). Quantitative real-time PCR analyzed MAGEA2 in U87, U251, D373MG and D409MG cells stably transfected with EGFP-AJAP1 or EGFP-N1 plasmid to validate the microarray findings. MAGEA2 expression was decreased in all AJAP1 positive glioma cell lines tested in triplicate (Fig. 1B), as demonstrated by western blot analysis (Fig. 1C).

Construction of the luciferase reporter plasmid with the MAGEA2 gene promoter. Promoter fragment $(1 \mathrm{~kb})$ of the MAGEA2 gene was amplified by PCR and inserted into a pGL3-enhancer vector to form the MAGEA2 promoter-luciferase reporter plasmid designated as $p G L 3-M A G E A 2$ (Fig. 2A). The recombinant plasmid was confirmed by double restriction enzyme digestion (Fig. 2B) and DNA sequence analysis. A significant increase (over 24 -fold greater, $\mathrm{P}<0.01$, Fig. $2 \mathrm{C}$ ) in relatively luciferase activity was observed in HEK293 cells co-transfected PGL3-MAGEA2 compared with vector control. Thus, the recombinant PGL3-MAGEA2 luciferase reporter plasmid was determined to be able to drive the luciferase expression.

AJAP1 transcriptional downregulated the expression of $M A G E A 2$. To explore the effects of AJAP1 on MAGEA2 gene expression, HEK293 cells were harvested $24 \mathrm{~h}$ after co-transfection with $p G L 3-M A G E A 2$ and EGFP-AJAP1. The control cells were co-transfected with $p G L 3-M A G E A 2$ and GFP-N1 plasmid. The MAGEA2 promoter activity was considerably blocked $(78.7 \%, \mathrm{P}<0.01)$ in HEK293 cells with GFP-AJAP1 co-transfection, compared with control cells (Fig. 3A). To further confirm the transcriptional relation- 
A

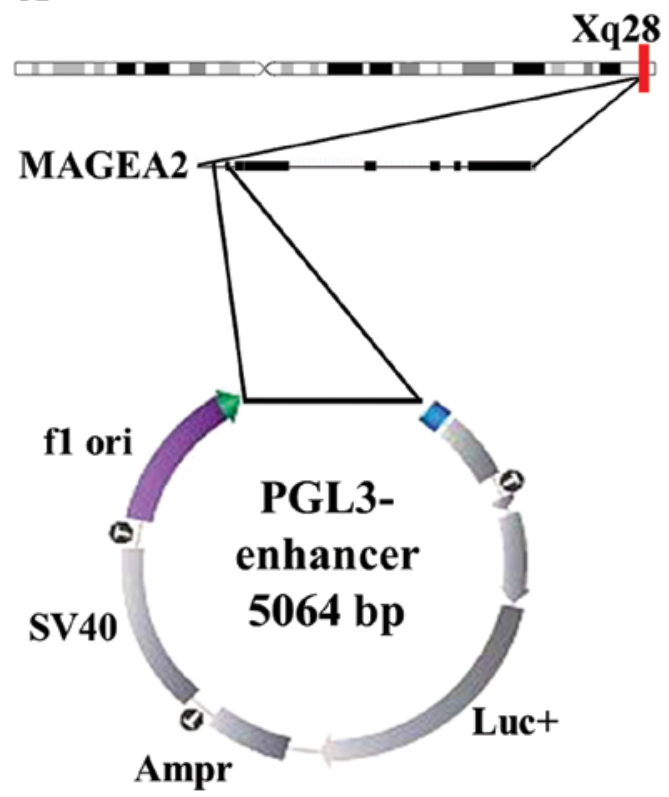

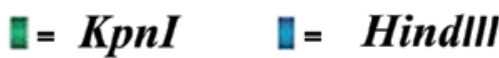

B

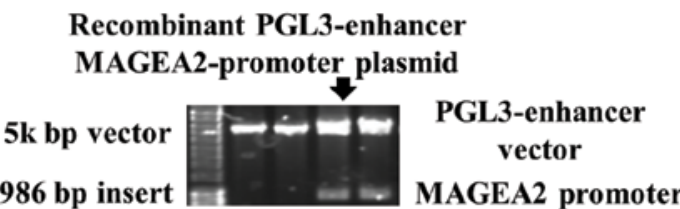

C

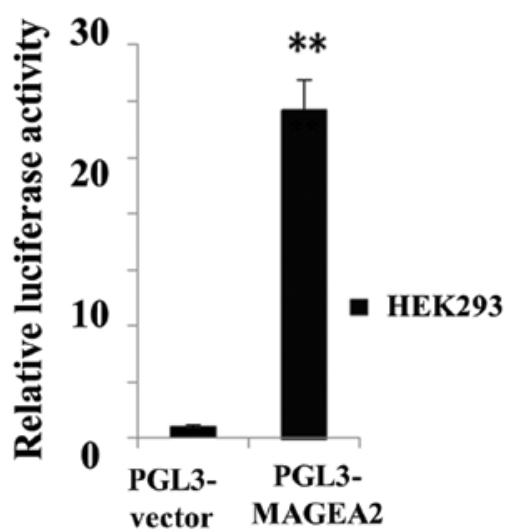

Figure 2. Construction and confirmation of the luciferase reporter plasmid with the MAGEA2 gene promoter. (A) Schematic figure of PGL3-enhancer-MAGEA2promotor plasmid construction. The promoter fragment of the MAGEA2 gene (map to Xq28) was amplified by PCR using human genomic DNA as the template. The forward primer was designed with a KpnI site at the 5'-end and the reverse primer with a HindIII site at the 5'-end. The amplified fragment was isolated and purified following digesting with KpnI and HindIII, then ligated into the equivalent sites of the pGL3-enhancer vector to generate the pGL3-MAGEA2 construct. (B) Electrophoresis of double digestion (KpnI and HindIII) recombinant plasmid and control (PGL3-enhancer) verified the luciferase reporter plasmid contained the MAGEA2 gene promoter. (C) PGL3-enhancer-vector plasmids (1.0 $\mu \mathrm{g})$ or control (PGL3-MAGEA2) plasmids (1.0 $\mu \mathrm{g})$ were transiently co-transfected with PRL-CMV (0.1 ng). Twenty-four hours after transfection, subsequently assayed for dual luciferase activity. The activities of firefly luciferase in pGL3-constructs and Renilla luciferase in pRL-CMV were determined by the dual-luciferase reporter assays. Luciferase activity is expressed as a ratio of firefly luciferase (PGL3) activity to Renilla activity (PRL-CMV). Data represent means \pm SE of luciferase activity of samples measured in triplicate for 3 independent experiments $\left({ }^{* *} \mathrm{P}<0.01\right.$; independent, two-tailed t-test).

A

$$
\text { }
$$

1.2

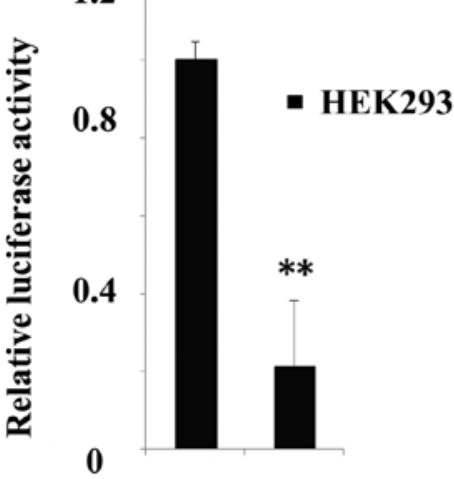

PGL3-MAGEA2 $+\quad+$

GFP-N1 +-

GFP-AJAP1
B

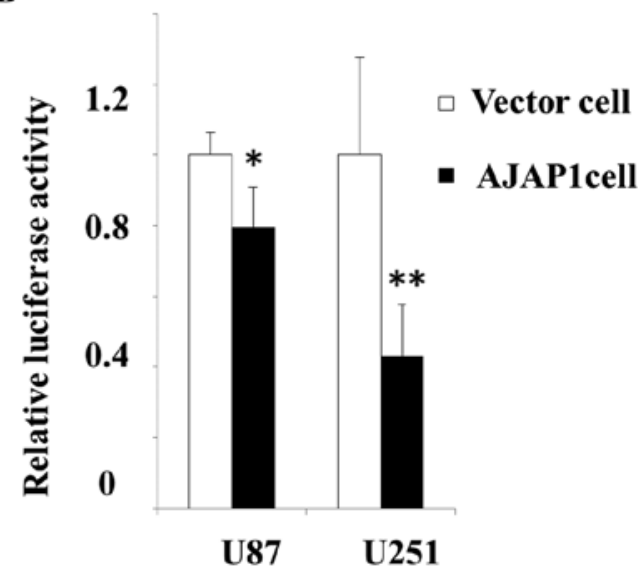

Figure 3. AJAP1 decreases activity of MAGEA2-promoter in HEK293, U87 and U251 glioma cells. (A) PGL3-MAGEA2 plasmids (0.5 $\mu \mathrm{g})$ were transiently co-transfected with EGFP-AJAP1 $(0.5 \mu \mathrm{g})$ or control (GFP-N1, $0.5 \mu \mathrm{g})$ and PRL-CMV $(0.1 \mathrm{ng})$ in HEK293 cells. Twenty-four hours after transfection, dual luciferase activity was measured. Luciferase activity is expressed as a ratio of firefly luciferase (PGL3) activity to Renilla activity (PRL-CMV). (B) PGL3-MAGEA2 plasmids $(1.0 \mu \mathrm{g})$ were transiently co-transfected with PRL-CMV $(0.1 \mathrm{ng})$ in U87 vector or AJAP1 cells and U251 vector or AJAP1 cells. Luciferase activity was assessed $24 \mathrm{~h}$ later and showed significant reduction in MAGEA2 promoter activity in the present of AJAP1. Data represent means \pm SE of luciferase activity of samples measured in triplicate for 3 independent experiments $\left({ }^{* *} \mathrm{P}<0.01,{ }^{*} \mathrm{P}<0.05\right.$; independent, two-tailed $\mathrm{t}$-test). 
A

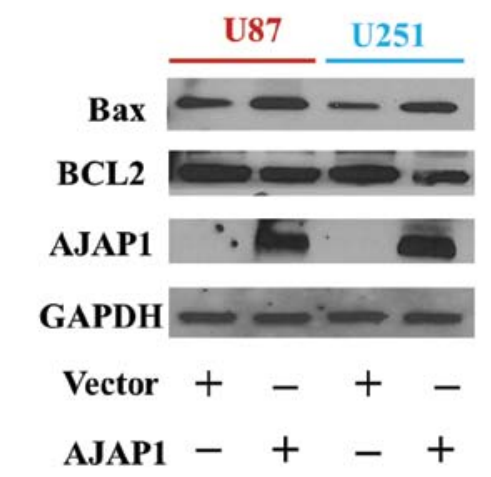

B

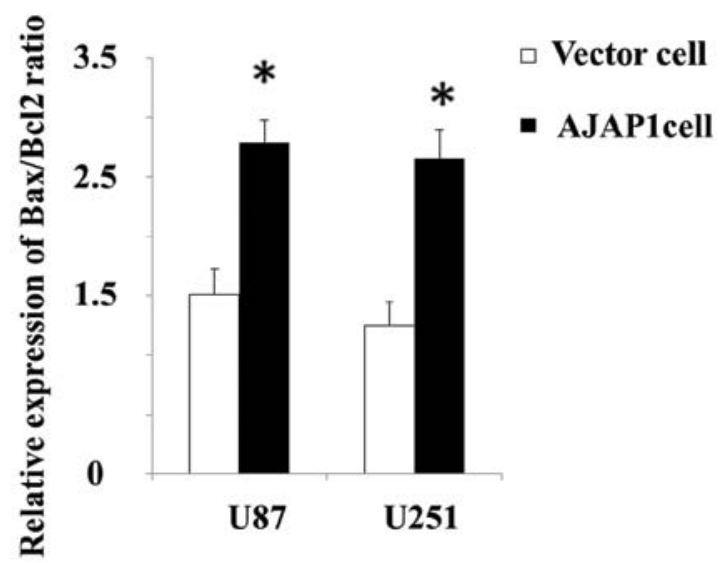

C

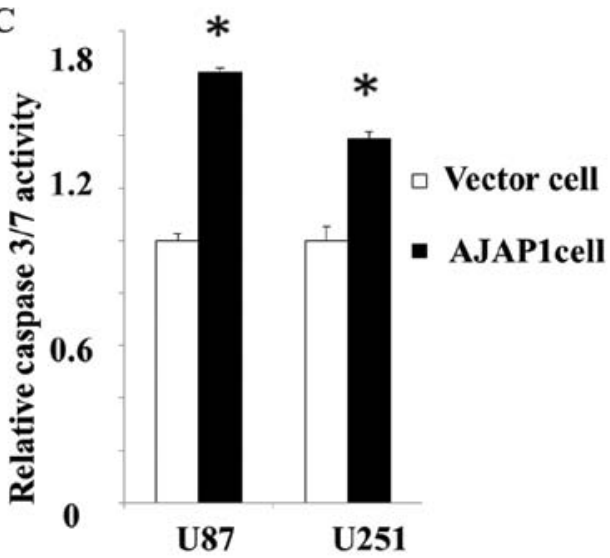

D
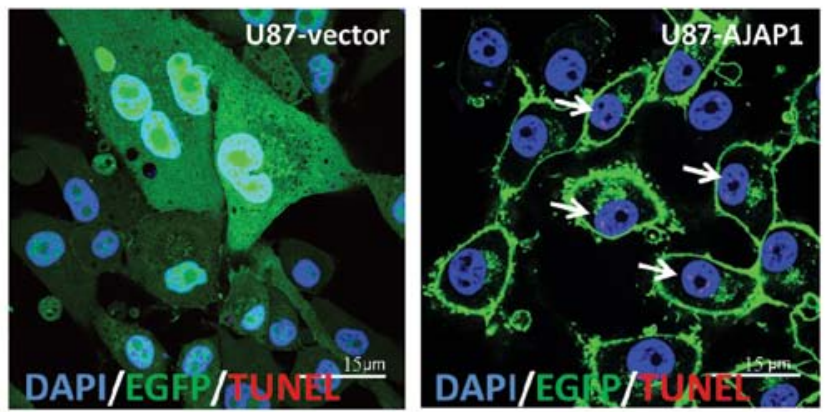

Figure 4. AJAP1 moderately induces apoptosis in GBM cells. (A) Representative western blot analysis of protein extracts for Bcl2, Bax and AJAP1 from $25 \mu \mathrm{g}$ of whole cell lysates with EGFP-AJAP1 transfected or control (EGFP-N1) in U87 MG cells and U251 MG cells. GAPDH was used as a loading control. (B) Histogram represents the relative expression of $\mathrm{Bax} / \mathrm{Bcl} 2$ ration calculated from each group. Data represent means $\pm \mathrm{SE}$ of apoptosis rate of samples counted in triplicate for 3 independent experiments. ( ${ }^{* *} \mathrm{P}<0.01,{ }^{*} \mathrm{P}<0.05$; independent, two-tailed t-test). (C) A total of 104 U87 MG cells and U251 MG cells expressing AJAP1 or control (EGFP-N1) were seeded into 96-well plates overnight. Caspase-3/7 activity was measured by Caspase-Glo 3/7 Reagent in vector and AJAP1 glioma cells. Histograms represent means $\pm \mathrm{SE}$ of caspase-3/7 activity (fold over control) of samples measured in triplicate for 3 independent experiments. ("P $<0.05$; independent, two-tailed t-test). (D) U87 vector or AJAP1 cells were plated in 24-well chamber slides (105 cells/well) and grown overnight. Cells were fixed, permeabilized and incubated with TUNEL reaction mixture overnight at $4^{\circ} \mathrm{C}$ followed by DAPI and mounted. Representative confocal microscopic fields of three independent experimental replicates are shown.

ship between AJAP1 and MAGEA2, glioma cell lines stable expressing AJAP1 gene or empty vector were transfected with pGL3-MAGEA2. We observed a significant decrease $(20.5 \%$ in U87 AJAP1 cells, $\mathrm{P}<0.05 ; 56.8 \%$ in U251 AJAP1 cells, $\mathrm{P}<0.05)$ in relative luciferase activity, compared with respective vector cells (Fig. 3B). These data support that expression of AJAP1 repressed MAGEA2 expression by physical interaction with the MAGEA2 gene promoter.

AJAP1 moderately induces cell apoptosis in GBM cells. The decrease in $\mathrm{Bax} / \mathrm{Bcl}-2$ ratio is thought to contribute to the resistance of glioma cells to therapy by modulating the apoptotic cascade (20). In this study, we measured the levels of Bax and Bcl-2 by western blot analysis to detect the putative pro-apoptosis function of AJAP1. We observed 39.6 and $42.2 \%$ increase in Bax/Bcl-2 ratio in cells overexpressing AJAP1, compared to the control cells $(\mathrm{P}<0.05)$ (Fig. 4A and $\mathrm{B})$. Caspase proteins are cysteine proteases that act downstream of the Bcl-2 family by initiating cellular breakdown during apoptosis (21). Among the effector caspases, caspase-3/7 is most frequently involved in tumor cellular apoptosis (22). To determine whether caspase$3 / 7$ is activated after AJAP1 involvement, caspase-3/7 activity was measured by the Caspase-Glo $3 / 7$ functional assay. In this study, results demonstrated a $34.1 \%$ increase $(\mathrm{P}<0.05)$ in U87 AJAP1 cells and $39.0 \%$ increase $(\mathrm{P}<0.05)$ in $\mathrm{U} 251$ AJAP1 cells, compared with vector cells (Fig. 4C). Labeling of degraded DNA by TUNEL suggests apoptotic mechanisms. Distribution of apoptotic nuclei in U87 cell lines showed a tendency towards an accumulation of apoptotic cells in the AJAP1 overexpressed group compared with vector control (Fig. 4D).

AJAP1 potentiates TMZ-induced cytotoxicity in GBM cells. To investigate the consequence of AJAP1 expression on the sensitivity to TMZ-induced cytotoxicity, the levels of Bax and Bcl-2 were measured by western blot analysis in all treatment groups. In this study, a dramatic increase in Bax/Bcl-2 ratio was observed in U87 and U251 cells exposed to AJAP1 overexpressed plus TMZ, compared to control cells not exposed to AJAP1 overexpression $(\mathrm{P}<0.01)$ (Fig. 5A). In AJAP1 overexpressed cells treated with TMZ, the caspase-3/7 activity was found dramatically increased compared with control group in U87 cells $(68.5 \%, \mathrm{P}<0.01)$ and U251 cells $(88.9 \%, \mathrm{P}<0.01)$ (Fig. 5B and C). TUNEL labeling showed that the U87 AJAP1 cells contain significantly more apoptotic nuclei $48 \mathrm{~h}$ after TMZ treatment compared with control 
A

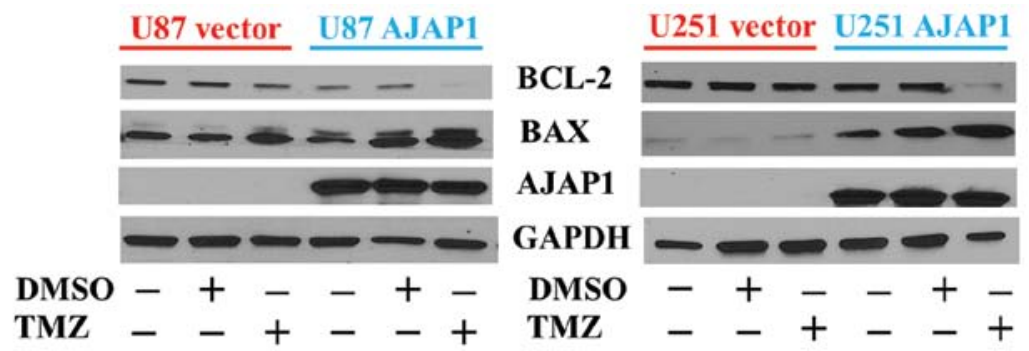

B

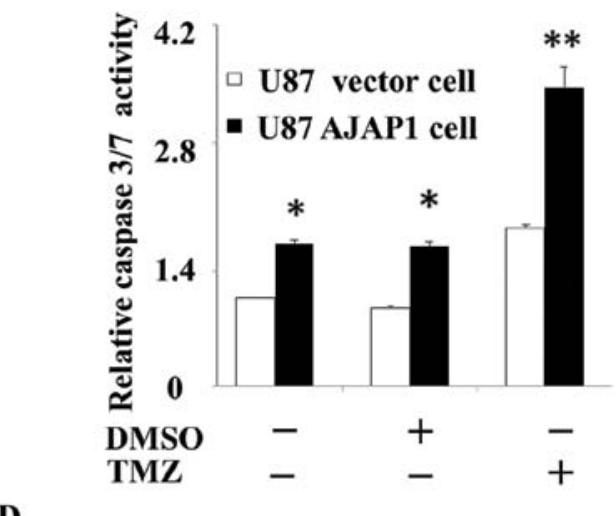

D
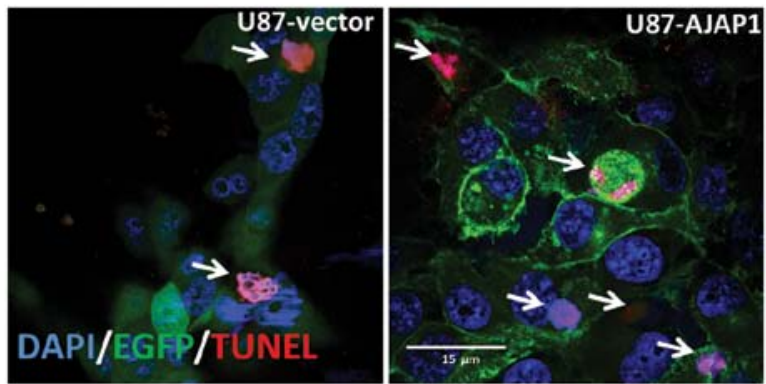

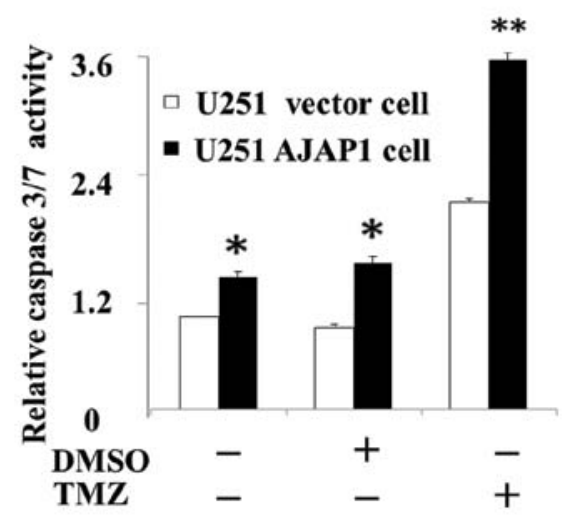

$\mathbf{E}$

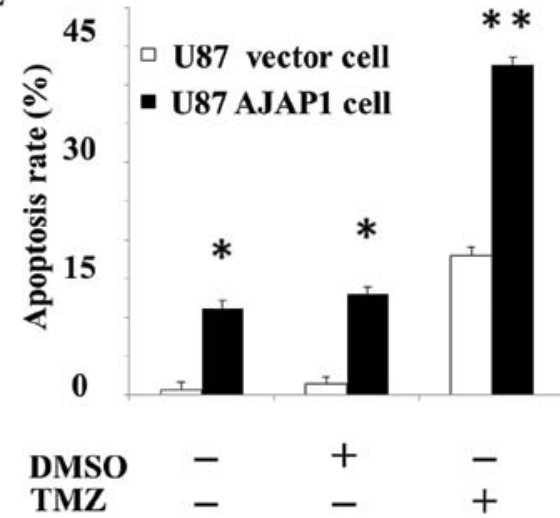

Figure 5. Restoration of AJAP1 increases cytotoxicity in U87 and U251 cells in the presence of TMZ $(100 \mu \mathrm{M})$ for 48 h. Control cells received DMSO (1:2,000) for $48 \mathrm{~h}$. (A) Representative western blot analysis of protein extracts for Bcl2, Bax and AJAP1. The expression of GAPDH was examined to ensure uniform protein loading in all lanes. (B and C) Apoptosis was determined by measuring caspase-3/7 activity in (B) U87 vector and AJAP1 cells and (C) U251 vector and AJAP1 cells as described. Data represent means \pm SE of caspase-3/7 activity (fold over control) of samples measured in triplicate for 3 independent experiments $\left({ }^{* *} \mathrm{P}<0.01,{ }^{*} \mathrm{P}<0.05\right.$; independent, two-tailed t-test). (D) U87 vector or AJAP1 cells were plated as previously described, then treated with $100 \mu \mathrm{M}$ TMZ for $48 \mathrm{~h}$. Fresh drug and medium were changed every $24 \mathrm{~h}$. Cells were fixed, permeabilized and incubated with TUNEL reaction mixture overnight at $4{ }^{\circ} \mathrm{C}$ followed by DAPI and mounted. Representative confocal microscopic fields of three independent experimental replicates are shown. (E) Histogram represents the percentage of apoptosis cells (TUNEL marked) calculated from each group. Data represent means \pm SE of apoptosis rate of samples counted in triplicate for 3 independent experiments. $\left({ }^{* *} \mathrm{P}<0.01,{ }^{*} \mathrm{P}<0.05\right.$; independent, two-tailed t-test).

group (36.7\%, P<0.01) (Fig. 5D and E). Moreover, U87 AJAP1 cells with TMZ treatment presented more typical morphological apoptotic changes, such as nuclear condensation, nuclear fragmentation and shrinkage of the cells (Fig. 5D). Taken together, these results showed that AJAP1 sensitized U87 and U251 cells to TMZ, as demonstrated by cellular Bax/Bcl-2 ratio, caspase3/7 activity and TUNEL labeling.

\section{Discussion}

The results summarized here strongly suggest that AJAP1 is a negative transcriptional regulator of MAGEA2, by possibly interacting with the promoter of MAGEA2 in GBM cells, resulting in downregulation of MAGEA2 expression by
mRNA and protein analyses. AJAP1 was initially described as a novel component of adherens junctions in polarized epithelial cells, which co-localized and co-immunoprecipitated with endogenous E-cadherin (23). Direct interaction between $\beta$-catenin and AJAP1 in an in vitro pull-down assay suggested that AJAP1 might be linked to the E-cadherin-mediated junctional complexes via $\beta$-catenin (24). $\beta$-catenin contains armadillo repeats and is able to bind to other proteins such as transcription factors (25). The ability of $\beta$-catenin to bind to other proteins is regulated by tyrosine kinases (26) and serine kinases such as GSK-3 (27). Various signals such as the Wnt signaling pathway can inhibit GSK-3-mediated phosphorylation of $\beta$-catenin, (28) allowing $\beta$-catenin to translocate to the cell nucleus, interact with transcription factors and 
regulate gene transcription. We hypothesize that AJAP1 may be translocated to the nucleus, possibly via its interaction with $\beta$-catenin complexes, where it then can bind and inhibit MAGEA2 transcription. The in-depth mechanism of how AJAP1 regulates MAGEA2 needs further investigation.

We previously demonstrated the frequent loss of AJAP1 in glioblastoma cell lines and primary tumors with high-resolution genome-wide mapping (29). AJAP1 is a putative tumor suppressor-like gene that maps to the 1 p36 region, where alterations are commonly associated with cancer (4) and has been implicated in cancer cell invasion, adhesion and migration (6,30-32). This study explores its potential role in gene regulation and cytotoxicity. Using extensive microarray analyses, we identified genetic alterations that occur in the context of glioma cell AJAP1 expression. We found that MAGEA2 expression is significantly decreased when AJAP1 is expressed. MAGEA2 provides a potent anti-apoptotic function to cancer cells partly through downregulation of the p53 pathway (14). Using in vitro approaches, we showed that AJAP1 expression produced proapoptotic results in glioma cells. Apoptosis is regulated by several protein families, including the upstream Bcl-2 family (i.e. the anti-apoptotic Bcl-2 and pro-apoptotic Bax) and the downstream caspase family (i.e. caspase-3/7) (33). Thus, we investigated the levels of expression of Bax and $\mathrm{Bcl}-2$ proteins in U87 and U251 GBM cells following restoration of AJAP1. Glioma cells with restoration of AJAP1 increase the expression of Bax and decrease the expression of $\mathrm{Bcl}-2$, which suggests a regulatory effect of AJAP1 in $\mathrm{Bax} / \mathrm{Bcl}-2$ ratios. $\mathrm{Bcl} 2$ is known to inhibit cytochrome $c$ release from mitochondria, thus blocking caspase activation, while Bax has the opposite function, which in turn promotes the release of cytochrome $c$ into the cytosol from mitochondria to activate caspase-3/7 (34). In our study, caspase-3/7 activity increased in the presence of AJAP1, compared with the vector control. These results indicated that AJAP1 could, at least in part, induce the mitochondria-related apoptosis pathways.

The resistance of GBM to standard of care chemotherapy (TMZ) may be more complex than simple dependence on MGMT levels $(35,36)$. It is likely a multifactorial phenomenon involving several major mechanisms, such as increased repair of DNA damage, reduced apoptosis, ectopic microRNA expression and increased energy-dependent efflux of chemotherapeutic drugs (37-39). Here, we show for the first time the enhancement of TMZ-induced apoptosis by AJAP1 in human glioma cell lines through MAGEA2 transcriptional regulation. Several studies have established that MAGE-A proteins confer resistance to chemotherapeutic drugs that act via p53-mediated apoptosis $(15,40,41)$. But the complete role of p53 in TMZ resistance is incompletely known. A trend toward increased TMZ sensitivity in patients with p53 mutations has also been suggested (39). We hypothesize that overexpression of AJAP1 in GBM may represent an alternative mechanism, aside from $\mathrm{p} 53$, to activate a tumor suppressive-like function of the p53 pathway.

In summary, AJAP1 appears to play a role in inducing apoptosis by directly downregulating MAGEA2 in GBM. In addition, AJAP1 can potentiate the sensitivity of TMZ in GBM cells. Therefore, AJAP1 may serve as a candidate biomarker for chemotherapy in GBM, but also a putative therapy opportunity for GBM.

\section{Acknowledgements}

We thank Dr Jing Mi (Department of Surgery, Duke University Medical Center) and Dr Hailan Piao (Department of Pathology, Duke University Medical Center) for expert technological assistance. This project was supported by Award no. I01BX007080 from the Biomedical Laboratory Research and Development Service of the VA Office of Research and Development.

\section{References}

1. Stupp R, Hegi ME, Mason WP, van den Bent MJ, Taphoorn MJ, et al: Effects of radiotherapy with concomitant and adjuvant temozolomide versus radiotherapy alone on survival in glioblastoma in a randomised phase III study: 5-year analysis of the EORTC-NCIC trial. Lancet Oncol 10: 459-466, 2009.

2. Gravendeel LA, Kouwenhoven MC, Gevaert O, de Rooi JJ, Stubbs AP, et al: Intrinsic gene expression profiles of gliomas are a better predictor of survival than histology. Cancer Res 69: 9065-9072, 2009.

3. Verhaak RG, Hoadley KA, Purdom E, Wang V, Qi Y, et al: Integrated genomic analysis identifies clinically relevant subtypes of glioblastoma characterized by abnormalities in PDGFRA, IDH1, EGFR, and NF1. Cancer Cell 17: 98-110, 2010.

4. Henrich KO, Schwab M and Westermann F: 1 p36 tumor suppression - a matter of dosage? Cancer Res 72: 6079-6088, 2012.

5. Zlatescu MC, TehraniYazdi A, Sasaki H, Megyesi JF, Betensky RA, Louis DN, et al: Tumor location and growth pattern correlate with genetic signature in oligodendroglial neoplasms. Cancer Res 61: 6713-6715, 2001.

6. Lin N, Di C, Bortoff K, Fu J, Truszkowski P, Killela P, Duncan C, McLendon R, Bigner D, Gregory S and Adamson DC: Deletion or epigenetic silencing of AJAP1 on 1p36 in glioblastoma. Mol Cancer Res 10: 208-217, 2012.

7. Aoki T, Hashimoto $\mathrm{N}$ and Matsutani M: Management of glioblastoma. Expert Opin Pharmacother 8: 3133-3146, 2007.

8. Stupp R, Mason WP, van den Bent MJ, Weller M, et al: Mirimanoff Radiotherapy plus concomitant and adjuvant temozolomide for glioblastoma. N Engl J Med 352: 987-996, 2005.

9. Salvati M, D'Elia A, Formichella AI and Frati A: Insights into pharmacotherapy of malignant glioma in adults. Expert Opin Pharmacother 10: 2279-2290, 2009.

10. Giese A, Bjerkvig R, Berens ME and Westphal M: Cost of migration: invasion of malignant gliomas and implications for treatment. J Clin Oncol 21: 1624-1636, 2003.

11. Kitange GJ, Carlson BL, Schroeder MA, Grogan PT, Lamont JD, Decker PA, Wu W, James CD and Sarkaria JN: Induction of MGMT expression is associated with temozolomide resistance in glioblastoma xenografts. Neuro Oncol 11: 281-289, 2009.

12. Frosina G: DNA repair and resistance of gliomas to chemotherapy and radiotherapy. Mol Cancer Res 7: 989-999, 2009.

13. Oliva CR, Nozell SE, Diers A, McClugage SG, et al: Acquisition of temozolomide chemoresistance in gliomas leads to remodeling of mitochondrial electron transport chain. J Biol Chem 285: 39759-39767, 2010.

14. Marcar L, Maclaine NJ, Hupp TR and Meek DW: Mage-A cancer/testis antigens inhibit p53 function by blocking its interaction with chromatin. Cancer Res 70: 10362-10370, 2010.

15. Monte M, Simonatto M, Peche LY, Bublik DR, Gobessi S, Pierotti MA, Rodolfo M and Schneider C: MAGE-A tumor antigens target p53 transactivation function through histone deacetylase recruitment and confer resistance to chemotherapeutic agents. Proc Natl Acad Sci 103: 11160-11165, 2006.

16. Yang B, O'Herrin SM, Wu J, Reagan-Shaw S, Ma Y, Bhat KM, Gravekamp C, Setaluri V, Peters N, Hoffmann FM, Peng H, Ivanov AV, Simpson AJ and Longley BJ: MAGE-A, MAGE-B and MAGE-C proteins form complexes with KAP1 and suppress p53-dependent apoptosis in MAGE-positive cell lines. Cancer Res 67: 9954-9962, 2007.

17. Bhan S, Negi SS, Shao C, Glazer CA, Chuang A, Gaykalova DA, Sun W, Sidransky D, Ha PK and Califano JA: BORIS binding to the promoters of cancer testis antigens, MAGEA2, MAGEA3, and MAGEA4, is associated with their transcriptional activation in lung cancer. Clin Cancer Res 17: 4267-4276, 2011. 
18. Glazer CA, Smith IM, Bhan S, Sun W, Chang SS, Pattani KM, Westra W, Khan Z and Califano JA: The role of MAGEA2 in head and neck cancer. Arch Otolaryngol Head Neck Surg 137: 286-293, 2011

19. Nardiello T, Jungbluth AA, Mei A, Diliberto M, Huang X, Dabrowski A, Andrade VC, Wasserstrum R, Ely S, Niesvizky R, Pearse R, Coleman M, Jayabalan DS, Bhardwaj N, Old LJ, Chen-Kiang S and Cho HJ: MAGE-A inhibits apoptosis in proliferating myeloma cells through repression of Bax and maintenance of survivin. Clin Cancer Res 17: 4309-4319, 2011

20. Sawada M, Nakashima S, Banno Y, Yamakawa H, Hayashi K, Takenaka K, Nishimura Y, Sakai N and Nozawa Y: Ordering of ceramide formation, caspase activation, and $\mathrm{Bax} / \mathrm{Bcl}-2$ expression during etoposide-induced apoptosis in C6 glioma cells. Cell Death Differ 7: 761-772, 2000.

21. Tanaka K, Asanuma M and Ogawa N: Molecular basis of anti-apoptotic effect of immunophilin ligands on hydrogen peroxide-induced apoptosis in human glioma cells. Neurochem Res 29: 1529-1536, 2004.

22. Stegh AH, Chin L, Louis DN and DePinho RA: What drives intense apoptosis resistance and propensity for necrosis in glioblastoma? A role for Bcl2L12 as a multifunctional cell death regulator. Cell Cycle 7: 2833-2839, 2008

23. Shore EM and Nelson WJ: Biosynthesis of the cell adhesion molecule uvomorulin (E-cadherin) in Madin-Darby canine kidney epithelial cells. J Biol Chem 266: 19672-19680, 1991.

24. Bharti S, Handrow-Metzmacher H, Zickenheiner S, Zeitvogel A, Baumann R and Starzinski-Powitz A: Novel membrane protein shrew-1 targets to cadherin-mediated junctions in polarized epithelial cells. Mol Biol Cell 15: 397-406, 2004.

25. Gottardi CJ and Peifer M: Terminal regions of beta-catenin come into view. Structure 16: 336-338, 2008.

26. Lilien J and Balsamo J: The regulation of cadherin-mediated adhesion by tyrosine phosphorylation/dephosphorylation of $\beta$-catenin. Curr Opin Cell Biol 17: 459-465, 2005.

27. Castellone MD, Teramoto H, Williams BO, Druey KM and Gutkind JS: Prostaglandin E2 promotes colon cancer cell growth through a Gs-axin-beta-catenin signaling axis. Science 310: 1504-1510, 2005

28. Liu X, Rubin JS and Kimmel AR: Rapid Wnt-induced changes in GSK3beta associations that regulate beta-catenin stabilization are mediated by Galpha proteins. Curr Biol 15 1989-1997, 2005.

29. Hino S, Tanji C, Nakayama KI and Kikuchi A: Phosphorylation of $\beta$-catenin by cyclic AMP-dependent protein kinase stabilizes $\beta$-catenin through inhibition of its ubiquitination. Mol Cell 25: 9063-9072, 2005.
30. McDonald JM, Dunlap S, Cogdell D, et al: The SHREW1 gene, frequently deleted in oligodendrogliomas, functions to inhibit cell adhesion and migration. Cancer Biol Ther 5: 300-304, 2006.

31. Schreiner A, Ruonala M, Jakob V, et al: Junction protein shrew-1 influences cell invasion and interacts with invasion promoting protein CD147. Mol Biol Cell 18: 1272-1281, 2007.

32. Cogdell D, Chung W, Liu Y, McDonald JM, Aldape K, Issa JP, Fuller GN and Zhang W: Tumor-associated methylation of the putative tumor suppressor AJAP1 gene and association between decreased AJAP1 expression and shorter survival in patients with glioma. Chin J Cancer 30: 247-253, 2011.

33. Jarskog LF, Selinger ES, Lieberman JA and Gilmore JH: Apoptotic proteins in the temporal cortex in schizophrenia: high bax/bcl-2 ratio without caspase-3 activation. Am J Psychiatry 161: 109-115, 2004.

34. Ryan KM, Phillips AC and Vousden KH: Regulation and function of the $\mathrm{p} 53$ tumor suppressor protein. Curr Opin Cell Biol 13: 332-337, 2001.

35. Kaina B and Christmann M: DNA repair in resistance to alkylating anticancer drugs. Int J Clin Pharmacol Ther 40: 354-367, 2002.

36. Bredel M, Bredel C, Juric D, et al: Tumor necrosis factoralpha induced protein 3 as a putative regulator of nuclear factorkappaB- mediated resistance to O6-alkylating agents in human glioblastoma. J Clin Oncol 24: 274-287, 2006.

37. Prokopenko $\mathrm{O}$ and Mirochnitchenko O: Ischemia-reperfusioninducible protein modulates cell sensitivity to anticancer drugs by regulating activity of efflux transporter. Am J Physiol Cell Physiol 296: 1086-1097, 2009.

38. Chekhun VF, Lukyanova NY, Kovalchuk, et al: Epigenetic profiling of multidrug-resistant human MCF-7 breast adenocarcinoma cells reveals novel hyper- and hypomethylated targets. Mol Cancer Ther 6: 1089-1098, 2007.

39. Roberti A, Sala DL and Cinti C: Multiple genetic and epigenetic interacting mechanisms contribute to clonally selection of drug-resistant tumors: current views and new therapeutic prospective. J Cell Physiol 207: 571-581, 2006.

40. Duan Z, Duan Y, Lamendola, DE, Yusuf RZ, et al: Overexpression of MAGE/GAGE genes in paclitaxel/doxorubicin resistant human cancer cell lines. Clin Cancer Res 9: 2778-2785, 2003.

41. Weeraratne SD, Amani V, Neiss A, et al: MiR-34a confers chemosensitivity through modulation of MAGE-A and p53 in medulloblastoma. Neuro Oncol 13: 165-175, 2011. 